\title{
Image Processing and Quantification in FIB Reconstructions. Tin Whisker and Hillock Case Study.
}

\author{
M.P. Marsh, ${ }^{*}$ M.E. Williams, ${ }^{* *} \mathrm{~K}-\mathrm{W}$ Moon, ${ }^{* *}$ and W.J. Boettinger** \\ *Visualization Sciences Group, Burlington MA 01803 USA \\ **Metallurgy Division, NIST, Gaithersburg, MD 20899 USA
}

FIB imaging of tin (Sn) whiskers and hillocks is used to illustrate solutions to common image processing challenges and statistical analyses. Processing steps discussed here include 2D alignment of slices, shadow correction, image filtering, and high-throughput feature identification and segmentation. For statistical analysis, we demonstrate tools for computing measurements (e.g. surface area and volume) on the library of segmented features so that the distributions can be fully evaluated or used for subsequent processing steps.

The spontaneous growth of Sn whiskers on electronic components and circuit board assemblies at room temperature is a serious reliability problem for electronics. Numerous failures due to whiskerinduced short-circuiting have been reported, especially in high reliability hardware (e.g. aerospace, military, and medical device applications). Electronic component leads made of copper $(\mathrm{Cu})$ or $\mathrm{Cu}$ alloys are often coated with Sn by electrodeposition prior to use or storage to promote or preserve their solderability. Whisker growth was retarded in the past by the addition of small amounts of lead $(\mathrm{Pb})$ to the $\mathrm{Sn}$ electrodeposit. $\mathrm{Pb}$ usage has been discontinued because of environmental regulations.

Whiskers are currently understood to be caused by diffusion of Sn from the interior of the Sn coating along the complex 3D grain boundary network toward specific but sparse surface grains, which are pushed out of the deposit by accumulation of $\mathrm{Sn}$ at their base just beneath the surface [1]. Other surface eruptions and conical hillocks form by the same mechanism but are not a reliability threat because of their low aspect ratio. The diffusion is caused by compressive stress gradients that develop in the Sn due to the plating process itself and due to the reaction of the Sn with the underlying $\mathrm{Cu}$ layer to from $\mathrm{Cu}_{6} \mathrm{Sn}_{5}$. Characterization of the 3D Sn grain boundary network and the location and shape of the $\mathrm{Cu}_{6} \mathrm{Sn}_{5}$ particles beneath whiskers and hillocks will assist in the determination of what is unique about the particular Sn grain that develops into a potentially dangerous filament whisker and why it is different from the hundreds of surrounding Sn grains that remain dormant.

FIB excavation and imaging were performed at $50 \mathrm{~nm}$ increments with the Zeiss NVision 40 and Zeiss Neon 40 EsB SEM/FIB instruments. The gallium $(\mathrm{Ga})$ ion source was operated at $30 \mathrm{kV}$ and the secondary electron SEM images were acquired with the in-lens detector. Sn reacts with Ga at room temperature so we developed FIB techniques that minimize the Ga dose exposure on the cut face of the Sn electrodeposit. Significant contamination from redeposition can occur if milling is extended below the Sn layer into the $\mathrm{Cu}$ substrate.

Performing an accurate alignment is the first barrier to correctly interpreting the 3D reconstruction of slices. We employed Zeiss's predictive beam-shift during acquisition, which subsumes any need for 
stage adjustment. Further, we performed both an automated and manual alignment of the slice series, and find that the automated alignment is sufficient in many cases. The automated alignment routine uses iterative translational alignment of adjoining slices, by minimizing the linear leastsquares of each pair-wise image difference.

Signal shadowing results when objects lie in the path between the illuminated spot and the detector. When these objects occlude the signal (e.g. secondary electron emission), the signal from those positions is reduced. Despite the trapezoidal FIB trench, the trench floor causes significant shadowing, and progressive redeposition aggravates the problem. This shadowing changes the signal intensity in a spatially dependent way and confounds image interpretation. Our shadow correction procedure approximates the uneven illumination profile by downsampling with bi-cubic splines. This downsampled image is effectively a low-pass filtered image that is subsequently subtracted from the input image.

By combining median and diffusion filters, we were able to isolate and segment features of interest. We can identify the hillock, the surrounding grains, the $\mathrm{Cu}_{6} \mathrm{Sn}_{5}$ layer, and the $\mathrm{Cu}$ substrate. We demonstrate the image processing tools required to characterize the Sn hillock morphology, and plan future comparative work that will permit us to identify morphological hallmarks that can be specifically associated with Sn hillock and Sn whisker growth.

\section{Disclaimer}

Identification of commercial products does not imply recommendation or endorsement by the National Institute of Standards and Technology.

\section{References}

[1] W. J. Boettinger, C. E. Johnson, L. A. Bendersky, K.-W. Moon, M. E. Williams and G. R. Stafford, Acta Materialia, 53 (2005) 5033-5050.
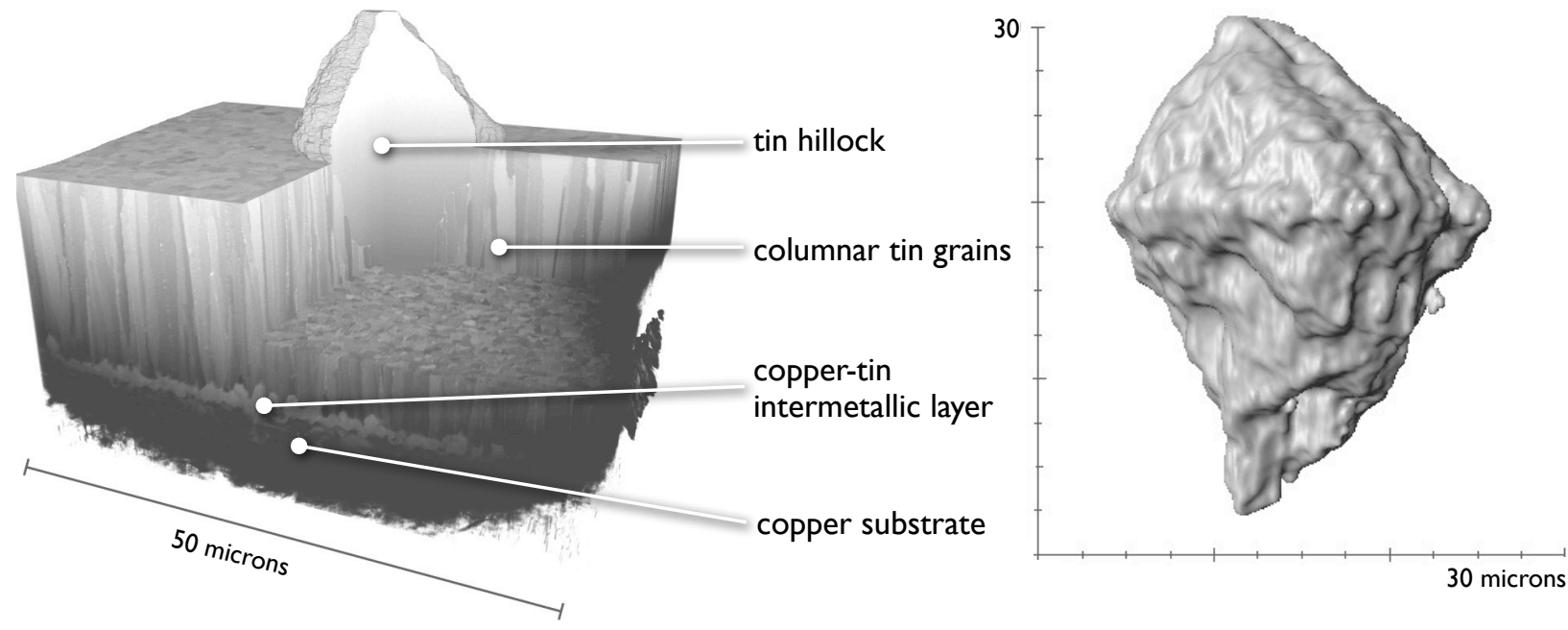

Figure 1. (Left) 3D View of aligned and masked reconstruction, with cutaway to reveal internal structures. (Right) Tin hillock, after segmentation and isolation from its $3 \mathrm{~d}$ context. 